\title{
Very compact radio emission from high-mass protostars
}

\section{Dust disks and ionized accretion flows}

\author{
F. F. S. van der Tak and K. M. Menten
}

\author{
Max-Planck-Institut für Radioastronomie, Auf dem Hügel 69, 53121 Bonn, Germany \\ e-mail: [vdtak;kmenten] @mpifr-bonn.mpg.de
}

Received 12 February 2005 / Accepted 22 March 2005

\begin{abstract}
This paper reports $43 \mathrm{GHz}$ imaging of the high-mass protostars W 33A, AFGL 2591 and NGC 7538 IRS9 at 0.04" and $\sim 0.6^{\prime \prime}$ resolution. In each case, weak ( mJy-level), compact $(\varnothing \sim 100 \mathrm{AU})$ emission is detected, which has an elongated shape (axis ratio $\sim 3$ ). However, for AFGL 2591 and NGC 7538 IRS9, the emission is single-peaked, while for the highestluminosity source, W 33A, a "mini-cluster" of three sources is detected. The derived sizes, flux densities, and broad-band radio spectra of the sources support recent models where the initial expansion of $\mathrm{H}$ II regions around very young O-type stars is prevented by stellar gravity. In these models, accretion flows onto high-mass stars originate in large-scale molecular envelopes and become ionized close to the star. These models reproduce our observations of ionized gas as well as the structure of the molecular envelopes of these sources on $10^{3}-10^{4} \mathrm{AU}$ scales derived previously from single-dish sub-millimeter continuum and line mapping. For AFGL 2591, the $43 \mathrm{GHz}$ flux density is also consistent with dust emission from a disk recently seen in near-infrared "speckle" images. However, the alignment of the $43 \mathrm{GHz}$ emission with the large-scale molecular outflow argues against an origin in a disk for AFGL 2591 and NGC 7538 IRS9. In contrast, the outflow from W 33A does not appear to be collimated. Together with previously presented case studies of W 3 IRS5 and AFGL 2136, our results indicate that the formation of stars and stellar clusters with luminosities up to $\sim 10^{5} L_{\odot}$ proceeds through accretion and produces collimated outflows as in the solar-type case, with the "additional feature" that the accretion flow becomes ionized close to the star. Above $\sim 10^{5} L_{\odot}$, clusters of $\mathrm{H}$ II regions appear, and outflows are no longer collimated, possibly as the result of mergers of protostars or pre-stellar cores.
\end{abstract}

Key words. accretion, accretion disks - stars: formation - instrumentation: high angular resolution - ISM: H II regions

\section{Introduction}

High-mass stars $\left(\gtrsim 10 M_{\odot}\right)$ play a major role in the evolution of their host galaxies, through strong ultraviolet radiation and winds, and through supernova explosions. However, the origin of high-mass stars is not well understood, due to the large distances $(\gtrsim \mathrm{kpc})$ and heavy extinctions $\left(A_{\mathrm{V}} \gtrsim 100\right)$ of the regions they form in. The optically visible main sequence life of OB-type stars is preceded by an "embedded" phase which lasts $\approx 15 \%$ of their lifetime (Churchwell 2002b). Observations at mid-infrared through radio wavelengths have shown that this embedded phase can be subdivided into several groups of objects: (1) Infrared dark clouds, where internal density maxima and temperature minima likely represent the initial conditions of high-mass star formation. (2) High-mass protostellar objects, where the central star is surrounded by a massive envelope with a centrally peaked temperature distribution. (3) Hot molecular cores, with large masses of warm and dense molecular gas, and large abundances of complex organic molecules evaporated off dust grains. (4) Ultracompact H II regions, where large pockets of ionized gas have developed, but stay confined to the stellar vicinity. (5) Compact, followed by classic $\mathrm{H}$ II regions, where the ionized gas expands hydrodynamically and disrupts the parental molecular cloud (for a review, see Garay \& Lizano 1999).

Despite this progress, key questions remain unanswered, which have been reviewed by Churchwell (2002a) and van der Tak (2003). The paramount question is whether highmass stars form via (disk) accretion like low-mass stars, or that other mechanisms such as coagulation of lower-mass stars or protostellar cores must be invoked (Bonnell et al. 1998). The ubiquity of massive bipolar outflows, detected in 21 out of a sample of 26 high-mass protostellar objects by Beuther et al. (2002) provides strong circumstantial evidence for disk accretion. The second central question of high-mass star formation is the relation with clustered star formation, i.e., the relation between the mass distribution of a star-forming region and its stellar density (e.g., Testi et al. 1999). Addressing these questions requires sub-arcsecond resolution, which at sub-millimeter wavelengths, with the Sub-Millimeter Array (SMA), is just coming within reach (Beuther et al. 2004). However, sub-arcsecond resolution can already be achieved at centimeter 
wavelengths, where two important diagnostic tools for highmass star formation are continuum emission from dense ionized gas and $\mathrm{H}_{2} \mathrm{O}$ maser emission.

Although the relation between the weak radio continuum emission detected in some high-mass protostars to their overall luminosity is presently not understood, it is clear that it marks a signpost for the exact position of the object. For example, in the case of Orion-KL, the weak radio source "I" almost certainly marks the position of the exciting object in the region, as the excitation of the surrounding $\mathrm{SiO}$ masers requires extreme temperatures and densities (Menten \& Reid 1995). Apart from the signpost effect, the detection of radio emission, which in the case of Orion-I is optically thick at frequencies up to at least $345 \mathrm{GHz}$, can also put constraints on the nature of the embedded protostar, in particular the accretion rate (Tan 2003).

Maser emission in the $\mathrm{H}_{2} \mathrm{O} 6_{16}-5_{23}$ line at $22.235 \mathrm{GHz}$ traces molecular gas with temperatures of $\sim 500 \mathrm{~K}$ and $n\left(\mathrm{H}_{2}\right) \sim$ $10^{9} \mathrm{~cm}^{-3}$ (Neufeld \& Melnick 1991) as occur for instance in shocks. The high brightness of the maser emission permits high angular resolution techniques and makes it a powerful tool for studying gas motions on small scales.

We are exploring the diagnostic power of high-resolution cm-wave observations to study the formation of high-mass stars. In the source AFGL 2136 (Menten \& van der Tak 2004), weak, compact radio continuum emission was detected, as well as $\mathrm{H}_{2} \mathrm{O}$ maser emission which is redshifted from the systemic velocity by $\approx 4 \mathrm{~km} \mathrm{~s}^{-1}$. This emission may arise in clouds of infalling gas that are heated in accretion shocks. In another deeply embedded high-mass star-forming region, W3 IRS5, Claussen et al. (1994) and Tieftrunk et al. (1997) detected a cluster of seven compact radio continuum sources, some of which are transient. Combining highresolution millimeter-wave and mid-infrared observations of these sources, van der Tak et al. (2005) conclude that some of them are probably evaporating and/or shock-ionized clumps in the ambient material, while others, having mid-infrared counterparts, most likely contain young high-mass stars.

This paper presents new radio observations at high angular resolution of three deeply embedded regions of high-mass star formation. The sources are a subset of a sample of bright mid-infrared sources studied from the ground (Willner et al. 1982; Mitchell et al. 1990) and with ISO (Van Dishoeck 1998). Single-dish sub-millimeter continuum and molecular line mapping by van der Tak et al. (2000) showed massive envelopes with a centrally concentrated temperature and density structure. For the present radio continuum study, we selected four sources which cover a range of evolutionary states within the embedded phase, as indicated by their sub-millimeter and infrared spectra (van der Tak et al. 2003).

W 33A is a highly luminous object $\left(L=1 \times 10^{5} L_{\odot}\right)$ in the Galactic molecular ring. The kinematic distance based on CS and $\mathrm{C}^{34} \mathrm{~S}$ lines is $4 \mathrm{kpc}$ (van der Tak et al. 2000). Its massive, cold envelope $\left(\approx 1100 M_{\odot}\right.$ within $74000 \mathrm{AU}$ radius $)$ is a favourite target for observations of mid-infrared absorption features due to icy grain mantles (Gibb et al. 2000). The millimeter emission was resolved by van der Tak et al. (2000) into a double source with separation $5^{\prime \prime}$. SMA observations will be presented by Shirley et al. (in prep.). Two $\mathrm{H}_{2} \mathrm{O}$ maser spots are known, whose centroid lies 0.75 from the millimeter continuum peak at position angle $-49^{\circ}$ (Forster \& Caswell 1999). The maser velocities are redshifted by $0.2-1.5 \mathrm{~km} \mathrm{~s}^{-1}$ relative to the dense core.

AFGL 2591 is a relatively isolated luminous $(L=2 \times$ $10^{4} L_{\odot}$ ) protostar in the Cygnus $X$ region. Distances in this region range from 0.5 to $2 \mathrm{kpc}$; we adopt $d=1 \mathrm{kpc}$ and refer to van der Tak et al. (1999) for further discussion. The source is known for its very high velocity CO mid-infrared absorption (Mitchell et al. 1989). With an envelope mass of only $\approx 40 M_{\odot}$ within a $30000 \mathrm{AU}$ radius, it is probably the least embedded (most evolved) source studied here. A combination of compact radio continuum emission and $\mathrm{H}_{2} \mathrm{O}$ masers similar to AFGL 2136 has been observed by Trinidad et al. (2003). However, in AFGL 2591, the maser spots trace a helical structure in position-velocity space, probably due to a precessing outflow.

NGC 7538 IRS9 is a luminous $\left(L=4 \times 10^{4} L_{\odot}\right)$ source in the NGC 7538 region, which optical spectrophotometry puts at $d=2.8 \mathrm{kpc}$ (Crampton et al. 1978). This source combines strong mid-infrared absorption features by icy grain mantles with high velocity CO emission (Schutte et al. 1996; Mitchell \& Hasegawa 1991). The envelope mass of $\approx 430 \mathrm{M}_{\odot}$ inside a $66000 \mathrm{AU}$ radius makes it an intermediate object between W 33A and AFGL 2591 in terms of envelope evolution. Kameya et al. (1990) detected two $\mathrm{H}_{2} \mathrm{O}$ maser spots in NGC 7538 IRS9, which have their centroid 0.'54 separated from the millimeter continuum peak at position angle $40^{\circ}$. Their velocities are blueshifted by $4.7-17.1 \mathrm{~km} \mathrm{~s}^{-1}$ from the cloud velocity and they probably arise in the outflow.

\section{Observations}

Initial radio observations of W 33A, AFGL 2136, AFGL 2591, and NGC 7538 IRS9 were obtained on 2001 September 9, with the NRAO Very Large Array (VLA) ${ }^{1}$ in C-configuration. Twenty-six antennas observed the sources sequentially in standard continuum mode: two intermediate frequency (IF) pairs of width $50 \mathrm{MHz}$ each (43 MHz "effective" bandwidth) separated by $50 \mathrm{MHz}$. The central frequency of $43.3 \mathrm{GHz}$ is called $Q$-band by radio engineers. Calibration of the antenna gains and phases was done by "fast switching" to the sources 1817-254, 1829-106, $2013+370$, and $2248+439$ every $140 \mathrm{~s}$. The antenna pointing was checked every hour with a 1-min integration on the phase calibrators at a frequency of $8.4 \mathrm{GHz}$ (" $X$-band").

Follow-up observations were made on 2002 March 23 and 25, with the VLA in A-configuration. Twenty-three and twenty-four antennas produced useful data on these respective days. The observing procedure was the same as before, except that 1829-106 was used as phase calibrator for both W 33A and AFGL 2136.

The data were edited, calibrated and imaged with NRAO's Astronomical Image Processing System (AIPS). At the time

The National Radio Astronomy Observatory (NRAO) is operated by Associated Universities, Inc., under a cooperative agreement with the US National Science Foundation. 
of these observations, corrections for elevation-dependent antenna gain and atmospheric opacity were not applied automatically to VLA data, so we applied them ourselves. In addition, small corrections to the positions of some antennas were made.

The absolute flux density scale follows from observations of $1328+307$ (3C 286) which has a flux density of $1.46 \mathrm{Jy}$ at this frequency (Ott et al. 1994). For the phase calibrators we obtain flux densities of $0.49 \pm 0.01 \mathrm{Jy}$ for $1817-254,0.63 \pm$ $0.01 \mathrm{Jy}$ for $1829-106,2.70 \pm 0.06 \mathrm{Jy}$ for $2013+370$ and $0.28 \pm$ $0.01 \mathrm{Jy}$ for $2248+439$. In A-configuration, the primary flux calibrator, 3C 286, is resolved, so we did not use a point source model, but a detailed model (in the form of Clean components) supplied by the VLA staff. We find flux densities of $0.71 \pm$ $0.02 \mathrm{Jy}$ for $1829-106,3.61 \pm 0.12 \mathrm{Jy}$ for $2013+370$ and $0.29 \pm$ $0.01 \mathrm{Jy}$ for $2248+439$.

Figure 1 shows the C-array maps, obtained by a Fourier transform of the $u v$ data with uniform weighting and deconvolution with the Clean algorithm. These maps have rms noise levels of $0.23 \mathrm{mJy} /$ beam. The synthesized beam sizes (FWHM) are $0.84 \times 0.41$ arcsec at position angle $(\mathrm{PA})-20^{\circ}$ for $\mathrm{W} 33 \mathrm{~A}$, $0.53 \times 0.41$ arcsec at PA $-44^{\circ}$ for AFGL 2591, and $0.55 \times$ 0.42 arcsec at $\mathrm{PA}-19^{\circ}$ for NGC 7538 IRS9. Figure 2 shows the A-array maps, which have rms noise levels of $0.18 \mathrm{mJy} / \mathrm{beam}$. Restoring beam axes are $78 \times 36$ mas at PA $-28^{\circ}$ for W $33 \mathrm{~A}$, $43 \times 37$ mas at PA $-36^{\circ}$ for AFGL 2591 , and $47 \times 36$ mas at PA $-42^{\circ}$ for NGC 7538 IRS9. Note that the regions shown in Fig. 2 are about the size of the compact emission sources from Fig. 1.

\section{Results}

\subsection{Positions and sizes}

Most images show single point sources. Only the C-array map of AFGL 2591 has an additional nearby extended H II region. This source was seen before by Campbell (1984) at $4.9 \mathrm{GHz}$ and by van der Tak et al. (1999) at $86 \mathrm{GHz}$, and appears partially resolved in our data. The flux density in a box around this source is $67.6 \mathrm{mJy}$; the source is presumably resolved out in A-array. We do not detect the somewhat extended source VLA2, which Trinidad et al. (2003) only detect at frequencies below $10 \mathrm{GHz}$. This source may be of non-thermal nature, or resolved out.

Table 1 presents the results of Gaussian fits to the observed compact radio emission, obtained with the task JMFIT in AIPS. The A-array map of W 33A may show two weak sources in addition to the source seen with the $\mathrm{C}$-array. We call the $43 \mathrm{GHz}$ sources in $\mathrm{W} 33 \mathrm{~A} \mathrm{Q} 1, \mathrm{Q} 2$ and Q3, in order of decreasing brightness. Figure 3 shows close-ups of these sources. If sources Q2 and Q3 are confirmed by more sensitive observations, W 33A may resemble the W3 IRS5 region, where a cluster of radio sources exists, some of which are internally, others externally ionized (van der Tak et al. 2005).

The position of $\mathrm{W} 33 \mathrm{~A}$ Q1 coincides with the $86 \mathrm{GHz}$ source MM1 from van der Tak et al. (2000). The A-array image also shows an extension of the emission toward the southwest, which was also seen at $86 \mathrm{GHz}$ and called MM2. Our data do not clearly show a second peak, however. The $\mathrm{H}_{2} \mathrm{O}$ maser
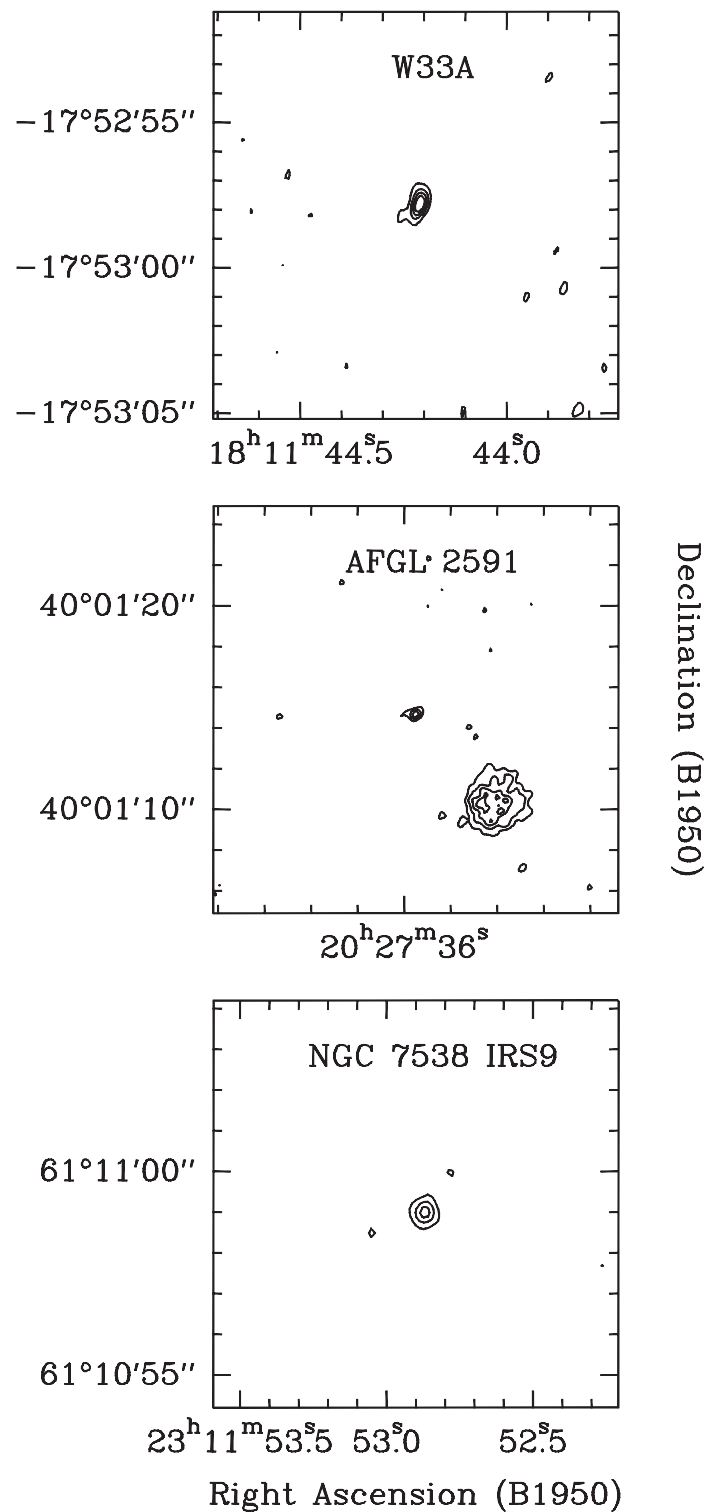

Fig. 1. VLA C-array $43 \mathrm{GHz}$ images of our targets. Contours start at $0.6 \mathrm{mJy} /$ beam and increase by $0.6 \mathrm{mJy} / \mathrm{beam}$ for $\mathrm{W} 33 \mathrm{~A}$ (top); for AFGL 2591 (middle), contours start at 0.7 and increase by $0.7 \mathrm{mJy} /$ beam; for NGC 7538 IRS9 (bottom), contours start at 0.5 and increase by $1.0 \mathrm{mJy} /$ beam.

centroid (Forster \& Caswell 1999) lies 0.' 44 from the $43 \mathrm{GHz}$ peak, at position angle $-78^{\circ}$ (i.e., due West). The positions of AFGL 2591 and NGC 7538 IRS9 are consistent with the millimeter-wavelength (van der Tak et al. 1999, 2000) and midinfrared positions. The positions found in A-array are consistent with those found in C-array within their $2 \sigma$ errors.

The fitted FWHM sizes of the sources are in Cols. 6 and 7 , and the orientation in Col. 8 of Table 1 . The given sizes are deconvolved values, even though most sources are only resolved in one direction. In the few cases where no meaningful estimate of the minor axis could be obtained, we use the value for the major axis as upper limit. Using the distances from Sect. 1, the A-array sizes correspond to linear radii of $150 \mathrm{AU}$ for $\mathrm{W} 33 \mathrm{~A}$, 20 AU for AFGL 2591, and 55 AU for NGC 7538 IRS9. 

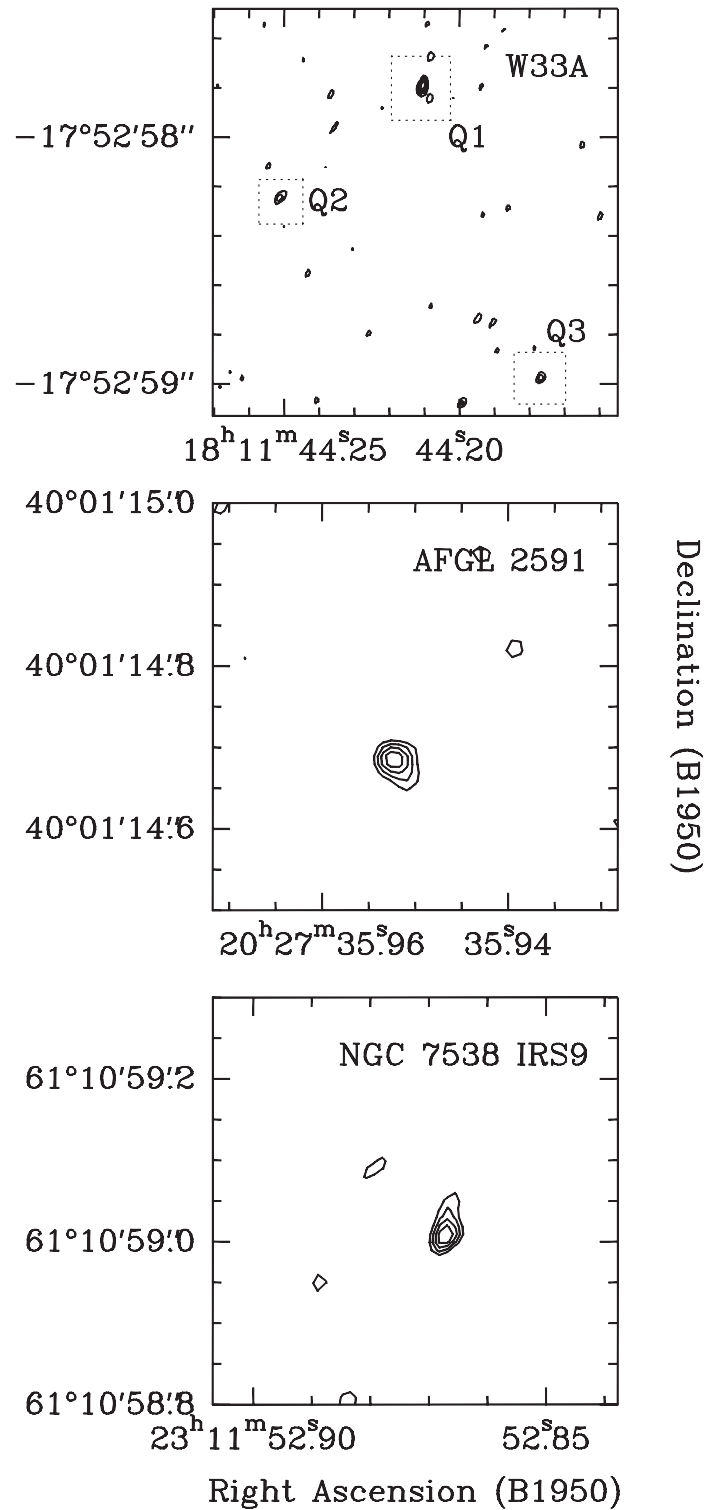

Fig. 2. VLA A-array $43 \mathrm{GHz}$ images of W 33A (top), AFGL 2591 (middle) and NGC 7538 IRS9 (bottom). Contours start at $0.45 \mathrm{mJy} /$ beam and increase by $0.15 \mathrm{mJy} / \mathrm{beam}$. The boxes in the W $33 \mathrm{~A}$ image are enlarged in the next figure.

The sizes found from the A-array data are not consistent with the C-array estimates, but factors of 5-7 smaller. This discrepancy suggests that the sources do not have a uniform brightness, but rather a centrally peaked brightness distribution. Each array picks up the "tip of the iceberg" at its own level of brightness sensitivity.

The derived axis ratios (or limits) of $\lesssim 0.5$ suggest a nonspherical, flattened geometry. The position angles found from the A-array images are consistent with those found from the $\mathrm{C}$-array images within their $2 \sigma$ errors. Combining the results from both array configurations, the orientation on the sky is $\mathrm{PA} \approx 20^{\circ}$ for $\mathrm{W} 33 \mathrm{~A} \mathrm{Q} 1, \mathrm{PA} \approx 73^{\circ}$ for AFGL 2591 and $\mathrm{PA} \approx 165^{\circ}$ for NGC 7538 IRS9.

\subsection{Flux densities and brightness temperatures}

Columns 4 and 5 of Table 1 give the peak intensities and the integrated flux densities of the sources. The flux densities determined from the A-array data are $\approx$ two times lower than those derived from the $\mathrm{C}$-array data. Since the phase calibrators are not systematically brighter in either configuration, we rule out a systematic calibration offset between the two data sets.

Sources can be weaker in more extended configurations because they are resolved, or because of increased phase noise on longer baselines. This "atmospheric decorrelation" decreases the visibility amplitudes by a factor of $\mathrm{e}^{-\sigma_{\phi}^{2} / 2}$, where $\sigma_{\phi}$ is the phase noise (in radians). From the phase noise in the calibrator data, we estimate a decorrelation by $\approx 5 \%$ in C-array and by $\approx 10 \%$ in A-array. Since the discrepancy is much larger than these factors, we conclude that decorrelation is unimportant here. Combined with the difference in observed sizes (Sect. 3.1), this discrepancy in flux density is additional evidence for a centrally peaked brightness distribution for these sources.

A first clue to the nature of the emission is the conversion of the measured flux densities and deconvolved sizes to brightness temperatures, using the Rayleigh-Jeans law. The total flux densities measured with the $\mathrm{C}$-array correspond to $T_{\mathrm{B}}=20-50 \mathrm{~K}$, while the values measured in A-array indicate $T_{\mathrm{B}}=190-850 \mathrm{~K}$. Most of these numbers are underestimates of the true brightness because the emission is not fully resolved, so we adopt $T_{\mathrm{B}}=500-1000 \mathrm{~K}$ as representative range for these sources.

If due to ionized gas, which has an intrinsic temperature of $10000 \mathrm{~K}$, the emission must be beam diluted, or optically thin, or both. However, the cm-wave spectra of W 33A and AFGL 2591 (Sect. 3.3) are too steep to be due to optically thin free-free emission. Therefore, if the observed emission is from ionized gas, the filling factor must be $\sim 0.05$. Using the source sizes derived in Sect. 3.1, this filling factor implies a linear radius of $\approx 30 \mathrm{AU}$ for $\mathrm{W} 33 \mathrm{~A}, \approx 5 \mathrm{AU}$ for AFGL 2591 and $\approx 10$ AU for NGC 7538 IRS 9 .

If the $43 \mathrm{GHz}$ emission is due to dust, the maximum physical temperature is $\approx 1500 \mathrm{~K}$, where dust sublimates. Therefore the emission must be close to optically thick and close to resolved. The mm-wave spectra (Sect. 3.3) indicate optically thick emission in W 33A and AFGL 2591, but optically thin emission in NGC 7538 IRS9. The filling factor must be at least $1 / 3$ and is probably close to unity, especially if the emitting dust is cooler than $1500 \mathrm{~K}$. The corresponding physical sizes assuming single condensations are 90-150 AU for W 33A, 5-20 AU for AFGL 2591, and 10-30 AU for NGC 7538 IRS 9.

\subsection{Radio spectra}

Table 2 contains radio flux densities for our three sources from the literature. Only data between frequencies of $3-300 \mathrm{GHz}$ and in beams $\lesssim 3^{\prime \prime}$ have been included. Figure 4 plots the data from Tables 1 and 2. We use the total flux densities measured in C-array, which are most comparable to the literature data. 
Table 1. Gaussian fits to the observed radio emission. Numbers in brackets are uncertainties in units of the last decimal.

\begin{tabular}{|c|c|c|c|c|c|c|c|}
\hline Source & $\begin{array}{l}\alpha(\mathrm{B} 1950) \\
\text { hh mm ss }\end{array}$ & $\begin{array}{l}\delta(\mathrm{B} 1950) \\
\circ, \prime \prime\end{array}$ & $\begin{array}{l}\text { Peak } I_{v} \\
\text { mJy/beam }\end{array}$ & $\begin{array}{l}\text { Total } S_{v} \\
\text { mJy }\end{array}$ & $\begin{array}{l}\text { Major axis } \\
\text { mas }\end{array}$ & $\begin{array}{l}\text { Minor axis } \\
\text { mas }\end{array}$ & $\begin{array}{l}\text { PA } \\
\text { deg }\end{array}$ \\
\hline \multicolumn{8}{|c|}{$C$-array } \\
\hline W 33A & $181144.2121(12)$ & $-175257.793(29)$ & $3.0(2)$ & $4.3(5)$ & $530(140)$ & $270(110)$ & $168(31)$ \\
\hline AFGL 2591 & $202735.9523(22)$ & +4001 14.694(17) & $2.6(2)$ & $3.3(5)$ & $440(110)$ & $<160$ & $89(15)$ \\
\hline NGC 7538 IRS9 & $231152.8696(14)$ & +611058.998(12) & $3.1(2)$ & $2.9(3)$ & $150(110)$ & $<250$ & $150(24)$ \\
\hline \multicolumn{8}{|c|}{ A-array } \\
\hline W 33A Q1 & $181144.2104(4)$ & $-175257.804(9)$ & $0.80(17)$ & $1.7(5)$ & $87(57)$ & $<67$ & $41(20)$ \\
\hline W 33A Q2 & $181144.2510(3)$ & $-175258.241(6)$ & $0.79(18)$ & $0.62(27)$ & $<49$ & $<12$ & $\ldots^{a}$ \\
\hline W 33A Q3 & $181144.1769(3)$ & $-175258.972(5)$ & $0.76(18)$ & $0.53(25)$ & $<37$ & $\ldots^{a}$ & $\ldots^{a}$ \\
\hline AFGL 2591 & $202735.9520(3)$ & +400114.682(4) & $0.95(16)$ & $1.9(5)$ & $50(15)$ & $29(23)$ & $33(39)$ \\
\hline NGC 7538 IRS9 & $231152.8671(3)$ & +61 $1059.017(4)$ & $1.1(2)$ & $1.1(3)$ & $40(17)$ &...$^{a}$ & $173(17)$ \\
\hline
\end{tabular}

The broad-band spectrum of W $33 \mathrm{~A}$ at frequencies up to $43 \mathrm{GHz}$ can be characterized by a spectral index $\gamma$, defined as

$S_{v} \propto v^{\gamma}$

with $\gamma=1.2 \pm 0.4$, while the spectrum steepens to $\gamma=2.3 \pm 0.2$ at higher frequencies. Similarly, for AFGL 2591, $\gamma=0.9 \pm 0.4$ at $v<43 \mathrm{GHz}$, while $\gamma=2.5 \pm 0.8$ at $v>43 \mathrm{GHz}$. The spectrum of NGC 7538 IRS9 is even steeper, $\gamma=3.0 \pm 0.5$, at $v>43 \mathrm{GHz}$, and inverted $(\gamma=-0.5 \pm 0.4)$ at centimeter wavelengths. The uncertainties in these spectral indices are dominated by the differences in values derived at different frequencies, rather than by the uncertainties of the individual flux densities (estimated as $10 \%$ for the VLA, $30 \%$ for OVRO at $86 \mathrm{GHz}$ and $50 \%$ at $230 \mathrm{GHz}$ ). The spectral indices may be affected by variability, since the measurements were not simultaneous. In the case of W3 IRS5, van der Tak et al. (2005) found radio variability by factors up to 3 .

The spectral indices of W 33A and AFGL 2591 indicate that the emission at $v \lesssim 43 \mathrm{GHz}$ is dominated by free-free emission, while the higher-frequency points are mostly due to dust emission. Similar conclusions hold for AFGL 2136 and Orion-I, which indeed have similar radio spectra (Menten \& van der Tak 2004).

The spectrum of NGC 7538 IRS9 differs from that of the other sources in two respects. First, the mm-wave spectrum is significantly steeper than $\gamma=2$, indicating that the dust emission in NGC 7538 IRS9 is optically thin. Second, NGC 7538 IRS9 has a "flattish" spectrum between 4.9 and $8.5 \mathrm{GHz}$, whose spectral index is consistent with 0 , but poorly determined, due to the limited signal-to-noise ratio in particular of the $8.5 \mathrm{GHz}$ emission (Sandell et al. 2005). The latter authors interpret this as free-free emission, which, given the spectral behaviour would have to be optically thin, which is unlikely for such a compact source. The sizes they determine for the radio source, $2^{\prime \prime} \times 1^{\prime \prime}$ at $8.46 \mathrm{GHz}$ and $0.7^{\prime \prime} \times 0.1^{\prime \prime}$ at $4.86 \mathrm{GHz}$, also are very uncertain, and should probably at best be considered as upper limits (consistent with our $43 \mathrm{GHz}$ data). We note however, that within the uncertainties, NGC 7538 IRS9's radio spectrum could be due to non-thermal emission, consistent with synchrotron emission from a jet, as seen before in the "Turner-Welch" object $\mathrm{W} 3\left(\mathrm{H}_{2} \mathrm{O}\right)$ by Reid et al. (1995). An origin of the radio emission in a jet is consistent with the source's morphology, which is unresolved in one dimension. Its orientation, almost north-south, is similar to that of the high-velocity $\mathrm{CO}$ outflow (Sandell et al. 2005), although we note that outflows around NGC 7538 IRS9 observed in other tracers $\left(\mathrm{H}_{2}\right.$ and $\left.\mathrm{HCO}^{+}\right)$ present a complex picture.

The centimeter-wave spectral indices of $\mathrm{W} 33 \mathrm{~A}$ and AFGL 2591 are intermediate between the values expected for optically thin $(\gamma=-0.1)$ and optically thick $(\gamma=2) \mathrm{H}$ II regions with a uniform temperature and density. An intermediate optical depth would lead to a "bent" spectrum which is not observed. However, the observed values of $\gamma \approx 1$ are expected in the case of $\mathrm{H}$ II regions with density gradients. The most plausible gradients are the cases of an ionized wind and an infalling envelope, which lead to $n \propto r^{-2}$ and $n \propto r^{-1.5}$ density structure, respectively. The expected spectral indices of $\gamma=0.6$ and $\gamma=0.1$ are somewhat below the observed values.

In the case of a power-law distribution of the electron density with radius, $n_{\mathrm{e}} \propto r^{-q}$, a spectral index of $\gamma=(2 q-3.1) /(q-$ 0.5 ) is expected (Olnon 1975). Therefore the observed value $\gamma \approx 1$ would indicate $q=2.6$. However, it is not clear which process would create and sustain such a steep density distribution. The sound speed of $\approx 10 \mathrm{~km} \mathrm{~s}^{-1}$ implies a crossing time of $\approx 50 \mathrm{yr}$ for our sources, so the process would have to act continuously. More plausible models will be discussed in Sect. 4.3.

\section{Discussion}

\subsection{Stellar ionization rates}

The flux densities $S_{v}$ in Table 1 can be combined with the distances $d$ from Sect. 1 to estimate the flux of Lyman continuum photons $N_{\mathrm{L}}$ emitted by the central star (e.g., Krügel 2003). In the optically thick limit, the "Strömgren" radius $r_{\mathrm{S}}$ of the 

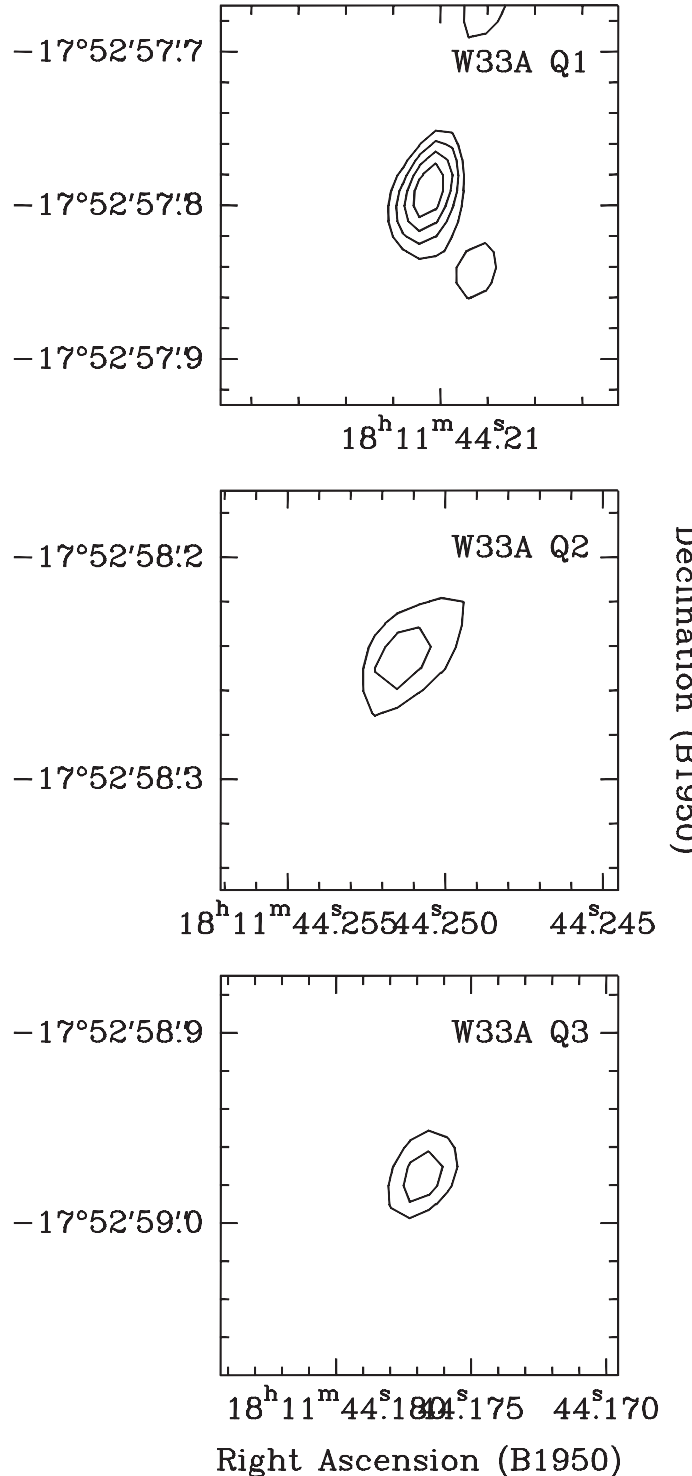

Fig. 3. Close-ups on the three $43 \mathrm{GHz}$ sources in our VLA A-array image of W 33A. Contour levels are as in previous figure.

$\mathrm{H}$ II region follows from blackbody emission at an assumed electron temperature of $T_{\mathrm{e}}=10^{4} \mathrm{~K}$ :

$S_{v}=B_{v}\left(T_{\mathrm{e}}\right) \frac{r_{\mathrm{S}}^{2}}{d^{2}} \frac{\pi}{\ln 2}$.

The derived radii of $33.5 \mathrm{AU}$ for $\mathrm{W} 33 \mathrm{~A}, 7.3 \mathrm{AU}$ for AFGL 2591, and 19.3 AU for NGC 7538 IRS9 are consistent with the observational limits (Sect. 3.1). To have a free-free optical depth of unity, the emission measure must be at least $8.4 \times 10^{9} \mathrm{~cm}^{-6} \mathrm{pc}$. These radii and emission measures together imply electron densities of $n_{\mathrm{e}}=5 \times 10^{6}$ to $1 \times 10^{7} \mathrm{~cm}^{-2}$. The ionizing photon flux then equals the recombination rate:

$N_{\mathrm{L}}=\frac{4 \pi}{3} r_{\mathrm{S}}^{3} n_{\mathrm{e}}^{2} \alpha_{\mathrm{B}}$

where $\alpha_{\mathrm{B}}$ is the "case B" recombination coefficient of $2.59 \times$ $10^{-13} \mathrm{~cm}^{3} \mathrm{~s}^{-1}$ (Osterbrock 1989). The values of $N_{\mathrm{L}}$ are $3.5 \times$ $10^{45} \mathrm{~s}^{-1}$ for $\mathrm{W} 33 \mathrm{~A}, 1.7 \times 10^{44} \mathrm{~s}^{-1}$ for AFGL 2591,
Table 2. Overview of literature data.

\begin{tabular}{lrrr}
\hline \hline Source & $\begin{array}{r}v \\
(\mathrm{GHz})\end{array}$ & $\begin{array}{r}S_{v} \\
(\mathrm{mJy})\end{array}$ & Reference \\
\hline W 33A & 8.4 & 0.8 & 4 \\
& 15 & 1.9 & 4 \\
& 86 & 24 & 5 \\
AFGL 2591 & 233 & 190 & 5 \\
& 4.9 & 0.4 & 1 \\
& 8.4 & 0.5 & 2 \\
& 22 & 1.6 & 6 \\
& 87 & 30 & 3 \\
NGC 7538 IRS9 & 226 & 151 & 3 \\
& 4.9 & 1.0 & 7 \\
& 8.5 & 0.8 & 7 \\
& 15 & $<0.5$ & 4 \\
& 107 & 43 & 5 \\
\hline
\end{tabular}

References: (1) Campbell (1984); (2) Tofani et al. (1995); (3) van der Tak et al. (1999); (4) Rengarajan \& Ho (1996); (5) van der Tak et al. (2000); (6) Trinidad et al. (2003); (7) Sandell et al. (2005).

and $1.2 \times 10^{45} \mathrm{~s}^{-1}$ for NGC 7538 IRS9. Values estimated using the optically thin limit,

$N_{\mathrm{L}}=7.54 \times 10^{46} \frac{S_{v}}{\mathrm{Jy}}\left(\frac{d}{\mathrm{kpc}}\right)^{2}\left(\frac{T_{\mathrm{e}}}{10^{4} \mathrm{~K}}\right)^{-0.45}\left(\frac{v}{\mathrm{GHz}}\right)^{-0.1}$

are within a factor of 2 from the thick estimates.

\subsection{Dust accretion rates}

Ionizing photon fluxes can also be estimated from the infrared luminosities, assuming that one single star dominates the Lyman continuum flux. We use the Geneva stellar structure models (Maeder \& Meynet 1989) to convert luminosities into stellar masses and effective temperatures, and the stellar atmosphere models by Schaerer \& de Koter (1997) to estimate Lyman continuum fluxes. The results are $M=28 M_{\odot}$, $T_{\text {eff }}=40000 \mathrm{~K}$ and $N_{\mathrm{L}}=8.5 \times 10^{48} \mathrm{~s}^{-1}$ for W 33A, $M=16 M_{\odot}$, $T_{\text {eff }}=33000 \mathrm{~K}$ and $N_{\mathrm{L}}=1.0 \times 10^{48} \mathrm{~s}^{-1}$ for AFGL 2591, and $M=20 M_{\odot}, T_{\text {eff }}=35000 \mathrm{~K}$ and $N_{\mathrm{L}}=2.2 \times 10^{48} \mathrm{~s}^{-1}$ for NGC 7538 IRS9.

These ionizing photon fluxes are factors of 2000-6000 higher than the estimates from the radio continuum emission (Sect. 4.1). Dust inside the H II region only accounts for factors of 2-3 absorption. One possibility is confinement by the ram pressure of accreting dust (cf. Walmsley 1995). The required accretion rate follows from equating the ram pressure to the thermal pressure of the $\mathrm{H}$ II region:

$\dot{M} v_{\text {ff }}=4 \pi r_{\mathrm{S}}^{2} n_{\mathrm{e}} k_{\mathrm{B}} T_{\mathrm{e}}$

where the free-fall velocity is given by

$v_{\mathrm{ff}}=\sqrt{G M_{*} / r_{\mathrm{S}}}$ 

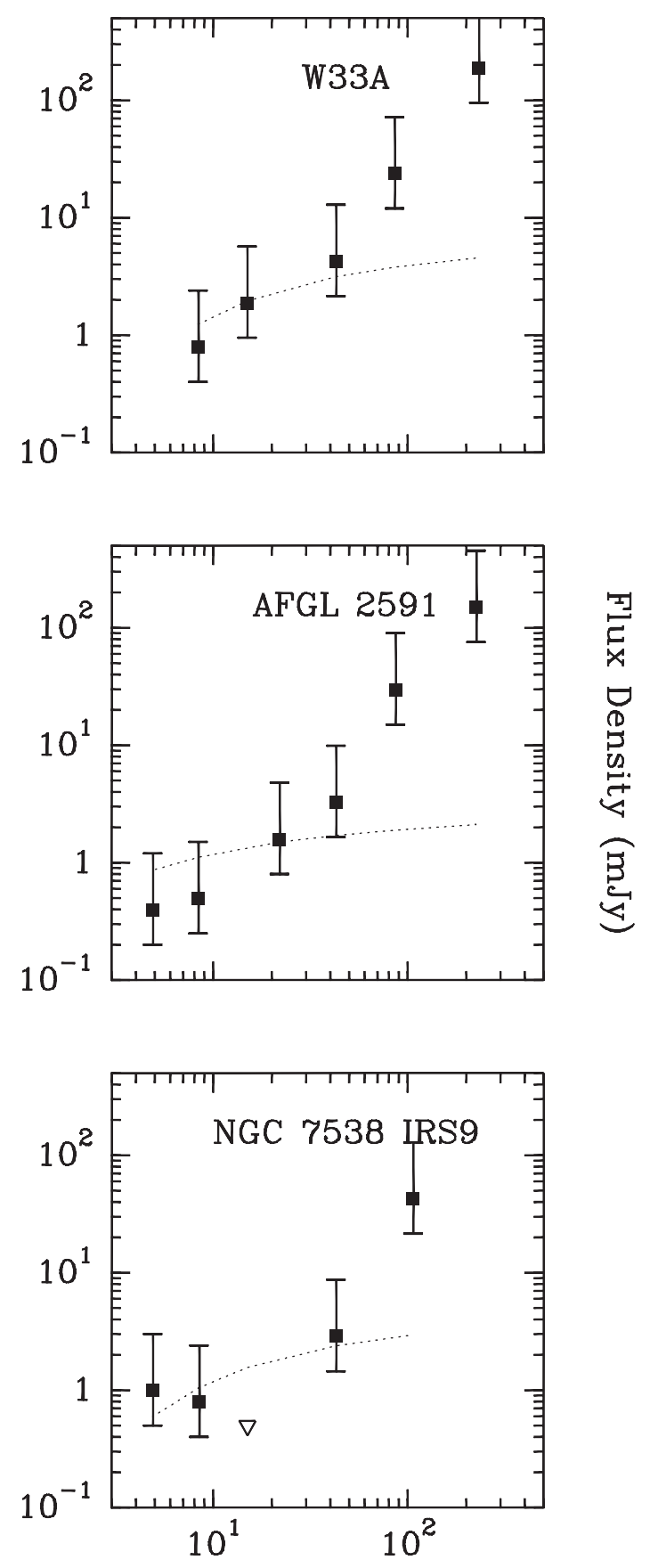

Frequency (GHz)

Fig. 4. Filled squares: Small-scale $\left(<3^{\prime \prime}\right)$ radio $(3-300 \mathrm{GHz})$ spectra of W 33A (top), AFGL 2591 (middle) and NGC 7538 IRS9 (bottom). The "ionized accretion flow" model from Sect. 4.3 (dotted lines) is seen to reproduce the emission up to $\sim 50 \mathrm{GHz}$ to within a factor of two. Flux densities at higher frequencies $>50 \mathrm{GHz}$ are dominated by dust emission, which the model does not consider.

which equals $42 \mathrm{~km} \mathrm{~s}^{-1}$ for $M_{*}=20 M_{\odot}$. With $r_{\mathrm{S}}=20 \mathrm{AU}$ and $n_{\mathrm{e}}=10^{7} \mathrm{~cm}^{-3}$, the estimated accretion rate is $6 \times$ $10^{-8} M_{\odot} \mathrm{yr}^{-1}$. This value is a lower limit because radiation pressure may slow down the dust from the free-fall speed, and because a stellar wind may add to the internal pressure.

\subsection{Ionized accretion flows}

Recent work by Keto (2002b) shows that stellar gravity prevents the hydrodynamical expansion of $\mathrm{H}$ II regions inside a "gravitational radius"

$r_{\mathrm{g}}=G M / 2 c_{\mathrm{s}}^{2}$

where $c_{\mathrm{S}}$ is the isothermal sound speed of ionized gas of $\approx 10 \mathrm{~km} \mathrm{~s}^{-1}$. This radius lies at $124 \mathrm{AU}$ for $\mathrm{W} 33 \mathrm{~A}, 71 \mathrm{AU}$ for AFGL 2591, and 89 AU for NGC 7538 IRS9. These values are comparable to the observational limits (Sect. 3.1).

In Keto's model, gas accreting onto the star is in molecular form at large radii, and becomes ionized at $r=r_{\mathrm{g}}$. The density structure of the molecular and ionized regions is given by the $n \propto r^{-1.5}$ free-fall profile. The molecular envelopes of our sources have similar or slightly flatter density structures (van der Tak et al. 2000).

The calculation of the radio spectrum of gravitationally bound H II regions is given by Keto (2003). Appendix A summarizes the derivation, with a few errors in the formulae corrected. The dotted lines in Fig. 4 are flux densities calculated using these corrected formulae.

The only free parameter in the calculation is $n_{0}$, the density at the molecular sonic point $r_{\mathrm{m}}$. Adopting $T=30 \mathrm{~K}$ as representative for the molecular gas, the location of its sonic point only depends on the mass of the central star. We have fitted the parameter $n_{0}$ to the observed (unweighted) flux densities of our sources at frequencies up to $43 \mathrm{GHz}$.

For W 33A, we find $n_{0}=3 \times 10^{5} \mathrm{~cm}^{-3}$ at $r_{\mathrm{m}}=0.49 \mathrm{pc}$, for AFGL 2591, we find $n_{0}=8 \times 10^{4} \mathrm{~cm}^{-3}$ at $r_{\mathrm{m}}=0.28 \mathrm{pc}$, and for NGC 7538 IRS9, we find $n_{0}=2 \times 10^{5} \mathrm{~cm}^{-3}$ at $r_{\mathrm{m}}=0.35 \mathrm{pc}$. These densities agree to factors of a few with the values derived from maps of sub-millimeter continuum and molecular lines (van der Tak et al. 2000).

Figure 4 shows that the model reproduces the observed flux densities to a factor of $\sim 2$, which is the expected uncertainty due to variability (in particular, variations in the accretion rate). We conclude that ionized accretion flows describe our data well, but that stronger tests are necessary to prove the model beyond doubt.

\subsection{Dust disks}

As an alternative model, we consider the suggestion by Preibisch et al. (2003) that the near-infrared emission from AFGL 2591 is the inner rim of a thick disk or envelope at the dust sublimation radius. These authors resolve the nearinfrared emission and derive a diameter of $40 \mathrm{AU}$, which is in good agreement with our measured $43 \mathrm{GHz}$ size, assuming a beam filling factor of unity. Since our measured $43 \mathrm{GHz}$ brightness is $850 \mathrm{~K}$ rather than $1500 \mathrm{~K}$, this interpretation requires either the optical depth to be $\approx 0.5$ at $43 \mathrm{GHz}$, or the dust temperature to be $\approx 850 \mathrm{~K}$, about half the sublimation temperature.

The luminosity of a $T=1500 \mathrm{~K}, 40 \mathrm{AU}$ diameter black body sphere is $80000 L_{\odot}$, which exceeds the observed value of $20000 L_{\odot}$ (Sect. 1). Therefore, if the object has this temperature and size, it can fill at most $1 / 4$ of the sky, and is likely a 
disk. However, if $T=850 \mathrm{~K}$, the spherical black body luminosity is only $9000 L_{\odot}$, within the observational limits.

The requirement of unit optical depth at $43 \mathrm{GHz}$ constrains the dust column density. Extrapolating Model 5 of the dust models by Ossenkopf \& Henning (1994), which provides a good fit to the mid- to far-infrared spectrum of AFGL 2591 (van der Tak et al. 1999), the $43 \mathrm{GHz}$ opacity is $0.06 \mathrm{~cm}^{2} \mathrm{~g}^{-1}$. The $2.2 \mu \mathrm{m}$ opacity is a factor of 80000 higher in this dust model, so that $\tau(43 \mathrm{GHz})=1$ implies $A_{\mathrm{V}}=890000$ or $N_{\mathrm{H}}=1.8 \times 10^{27} \mathrm{~cm}^{-2}$. This column density is orders of magnitude higher than measured for the envelopes of embedded highmass stars (van der Tak et al. 2000; Hatchell \& van der Tak 2003), but may be reached by massive circumstellar disks.

Another check of the disk model lies in the $2.2 \mu \mathrm{m}$ flux density of 2.8 Jy observed toward AFGL 2591 (e.g., Aitken et al. 1988). The compact component in the speckle observations contributes $\approx 60 \%$ of the $2.2 \mu \mathrm{m}$ flux density of AFGL 2591 in a $12^{\prime \prime}$ field (T. Preibisch, priv. comm.). If the emission is a black body at $T=1500 \mathrm{~K}$ with a radius $r=20 \mathrm{AU}$, matching the observed brightness requires a foreground $2.2 \mu \mathrm{m}$ extinction of $25 \mathrm{mag}$, or $20 \mathrm{mag}$ if $T=850 \mathrm{~K}$. The corresponding visual extinctions of 200-250 imply $N_{\mathrm{H}}=2.0-2.5 \times 10^{23} \mathrm{~cm}^{-2}$, which agrees very well with the $N\left(\mathrm{H}_{2}\right)=2.3 \times 10^{23} \mathrm{~cm}^{-2}$ measured in sub-millimeter emission (van der Tak et al. 1999). We conclude that the dust disk seen at $2.2 \mu \mathrm{m}$ by Preibisch et al. (2003) may also be responsible for our observed $43 \mathrm{GHz}$ emission. The evidence for this interpretation would be strengthened by measuring the orientation of the near-infrared emission, which the current speckle data do not constrain (T. Preibisch, priv. comm.).

\subsection{Relation with large-scale outflows}

The deconvolutions in Sect. 3.1 indicate flattened shapes for the radio sources, with axis ratios of $\approx 3$. One central prediction of disk accretion models of low-mass star formation is that the outflow axis is perpendicular to the disk plane. To see if such models may also work for high-mass star formation, this section compares the orientation of the $43 \mathrm{GHz}$ emission with that of the large-scale outflows observed in our sources.

It is difficult to constrain the outflow orientation of such objects from the commonly observed CO 1-0 line. The outflows appear poorly collimated in single-dish observations, and interferometric observations tend to resolve out most of the emission. For example, in AFGL 2591, one of the earliest known outflow sources, high velocity $\mathrm{CO}$ in the $1-0$ line is spread over a large area $\left(\sim 5^{\prime}\right.$ or $\left.\sim 1 \mathrm{pc}\right)$ and is poorly collimated (Lada et al. 1984). Better constraints come from higher- $J$ CO lines or other molecules such as $\mathrm{HCO}^{+}$(Beuther et al. 2002).

In the case of AFGL 2591, $\mathrm{CO} 3-2$ and $\mathrm{HCO}^{+}$4-3 mapping by Hasegawa \& Mitchell (1995) shows an outflow of size $90 \times 20^{\prime \prime}$, embedded in an arcminute-scale flow seen before in lower- $J$ CO lines. The East-West orientation agrees with the positions of Herbig-Haro objects and spots of shock-excited $\mathrm{H}_{2}$ emission. For NGC 7538 IRS9, maps of the CO 2-1 line (Mitchell et al. 1991) show an outflow with a North-South orientation $\left(\mathrm{PA} \sim 160^{\circ}\right.$ ). Very high velocities (up to $110 \mathrm{~km} \mathrm{~s}^{-1}$ ) are seen in the 3-2 line, but this emission is spatially unresolved (Mitchell \& Hasegawa 1991). For W 33A, outflow activity is demonstrated by the positions and velocities of $\mathrm{OH}$ and $\mathrm{H}_{2} \mathrm{O}$ masers (e.g., Fish et al. 2003) and by "wings" on the sub-millimeter emission line profiles of $\mathrm{CS}, \mathrm{HCO}^{+}$and other molecules (van der Tak et al. 2000, 2003). Unfortunately, there is no $\mathrm{CO}$ or $\mathrm{HCO}^{+}$map to give us the outflow orientation for this source. In fact, the outflow may not be bipolar, as $\mathrm{OH}$ masers usually appear in quasi-spherical outflows. Observations of $\mathrm{H}_{2} \mathrm{O}$ masers with 0.5 mas resolution have revealed spherical outflows from several other young high-mass stars (e.g., Torrelles et al. 2003).

The $43 \mathrm{GHz}$ emission in both AFGL 2591 and NGC 7538 IRS9 has a similar orientation on the sky as their large-scale outflows. This alignment argues against an origin of the $43 \mathrm{GHz}$ emission in a disk, and suggests an origin in a jet. However, thermal emission from an ionized jet such as seen in lower-mass objects (e.g., Trinidad et al. 2004) is inconsistent with the measured spectral indices (Sect. 3.3). Perhaps cool material entrained by the jet contains sufficient dust to produce the observed spectrum.

\subsection{Photo-evaporating disks}

Another potential source of our observed $43 \mathrm{GHz}$ emission is free-free emission from an ionized wind from a circumstellar disk that is being illuminated by the Lyman continuum of a hot central star. Such photo-evaporating disks have been described semi-analytically by Hollenbach et al. (1994) while numerical calculations were performed by Richling \& Yorke (1997). In these models, evaporation occurs in the outer disk, where the radiative force of the star is stronger than its gravitational force. In our case of a $\sim 20 M_{\odot}$ star, this radius occurs at $\sim 100 \mathrm{AU}$, which is comparable to our measured source size.

The models distinguish "strong" and "weak" stellar winds, depending on whether the ram pressure of the wind is comparable to the thermal pressure of the disk atmosphere or exceeds it. Wind speeds up to several $100 \mathrm{~km} \mathrm{~s}^{-1}$ have been measured in mid-infrared $\mathrm{CO}$ absorption and $\mathrm{H}$ emission lines toward AFGL 2591 and NGC 7538 IRS9 (Mitchell et al. 1991; Bunn et al. 1995) and may also apply to W 33A. For a wind speed of $500 \mathrm{~km} \mathrm{~s}^{-1}$, a stellar mass of $20 M_{\odot}$ and a Lyman continuum flux of $3 \times 10^{48} \mathrm{~s}^{-1}$ (Sect. 4.2), the critical mass loss rate is $2 \times 10^{-7} M_{\odot} \mathrm{yr}^{-1}$. The mass loss rates estimated from CO 2-1 are $\sim 10^{-4} M_{\odot} \mathrm{yr}^{-1}$, so the sources are clearly in the strong wind limit.

The predicted spectral shape of circumstellar disks that are being photo-evaporated by strong stellar winds is that of an optically thin $\mathrm{H}$ II region $(\gamma=-0.1)$ at short wavelengths, a thick $\mathrm{H}$ II region $(\gamma=2)$ at long wavelengths, and a "wind" spectrum $(\gamma=0.6)$ at intermediate wavelengths. For the high mass loss rates of our sources, the transition to optically thick emission lies at unobservably long wavelengths. The transition from $\gamma=0.6$ to $\gamma=-0.1$ is expected around $0.5 \mathrm{~cm}$ wavelength. However, the observed spectra have quite different shapes, so that the photo-evaporating disk model does not seem applicable to our sources. 


\subsection{Free electron absorption}

As a last option, we consider free-free absorption by $\mathrm{H}^{-}$as source of the observed $43 \mathrm{GHz}$ emission. This mechanism was originally proposed for the radio emission of Mira-type variable stars (Reid \& Menten 1997) and may play a role in Orion-KL (Beuther et al. 2004). The required free electrons come from photo-ionization of $\mathrm{Na}$ and $\mathrm{K}$ atoms, so that this mechanism only operates inside the dust sublimation radius.

Our observed brightness temperatures of $\sim 1500 \mathrm{~K}$ are consistent with this model, and combining the radii of $\sim 10 \mathrm{AU}$ with $N\left(\mathrm{H}_{2}\right) \sim 10^{27} \mathrm{~cm}^{-2}$ gives a density of $\sim 10^{13} \mathrm{~cm}^{-3}$, high enough to provide unit free electron opacity (Reid \& Menten 1997). The broad-band radio spectra of $\mathrm{W} 33 \mathrm{~A}$ and AFGL 2591 are not inconsistent with the predicted $\gamma=1.86$ behaviour if the flux densities are uncertain by a factor of $\sim 2$. For NGC 7538 IRS9, free electron absorption may explain the 15-107 GHz spectrum, while the lower-frequency data must have a different origin. In any case, stronger tests of this model will come from measurements of the density on $\sim 10$ AU scales.

\section{Conclusions}

We have detected weak, compact radio emission from three high-mass protostars which are deeply embedded in molecular envelopes. In two cases, the emission is probably due to bremsstrahlung from gravitationally bound $\mathrm{H}$ II regions. The derived densities are in good agreement with values found for the large-scale molecular envelope. Similar results were found for another object, W3 IRS5 (van der Tak et al. 2005), and additional support for accretion onto high-mass protostars comes from $\mathrm{H}_{2} \mathrm{O}$ maser mapping of AFGL 2136 (Menten \& van der Tak 2004). These findings support a picture of highmass star formation where the central star builds up mass by accretion from a molecular envelope. At small radii, the stellar Lyman continuum ionizes the accretion flow, but does not stop it. As the star gains mass, its surface temperature and ionizing flux increase. The circumstellar ionized region grows quasistatically until its radius reaches the critical value of $G M / 2 c_{\mathrm{s}}^{2}$. Stellar gravity is then unable to confine it, so that the $\mathrm{H}$ II region rapidly expands and halts the accretion.

However, this interpretation is not unique and may not be complete. For AFGL 2591, the data are also consistent with dust emission from a disk seen previously in the near-infrared. Furthermore, NGC 7538 IRS9 appears to have a non-thermal $\mathrm{cm}$-wave spectrum, such as produced by synchrotron emission from a jet. The mm-wave spectrum of this source is steeper than that of the other two, suggesting optically thin dust where the others are thick. In the case of $\mathrm{W} 33 \mathrm{~A}$, three radio continuum sources are seen at 3000-5000 AU separations. This "clustering" of radio sources resembles the W3 IRS5 region $\left(d=2.0 \mathrm{kpc}, L=1.4 \times 10^{5} L_{\odot}\right)$, where seven sources are found at about half the above separations (van der Tak et al. 2005). Follow-up VLA observations would be useful to find out if some of the $43 \mathrm{GHz}$ sources in $\mathrm{W} 33 \mathrm{~A}$ are transient, and mid-infrared imaging would reveal which of the three are self-luminous. Together with our previous "case studies" of AFGL 2136 (Menten \& van der Tak 2004) and W3 IRS5 (van der Tak et al. 2005), the current observations suggest that the formation of stars and stellar clusters with luminosities up to $\sim 10^{5} L_{\odot}$ proceeds through accretion and produces collimated outflows as in the case of solar-type stars. Such regions may well contain small star clusters (Testi et al. 1999) and clusters of non-thermal radio sources (Gómez et al. 2002). The main difference in the formation of $\sim 10^{4} L_{\odot}$ stars with that of solartype stars is that their accretion flows become ionized at small radii. In contrast, regions of $\sim 10^{5} L_{\odot}$ appear to produce clusters of $\mathrm{H}$ II regions and essentially uncollimated outflows, possibly as the result of mergers of protostars or pre-stellar cores.

In the future, the ionized accretion flow model should be tested by measuring the density profiles of hypercompact $\mathrm{H}$ II regions directly through radio continuum observations at $\sim 10$ AU resolution (10 mas at $1 \mathrm{kpc}$ ) and $\sim 0.1 \mathrm{mJy}$ sensitivity. The duration of the ionized accretion flow phase should be measured by a radio continuum survey of high-mass protostars at a resolution and sensitivity comparable to this work. Observations of radio recombination lines at high resolution exist for one source (Keto 2002a) and should be carried out for more. These efforts probably exceed the capabilities of the VLA, but will be feasible with the e-VLA (after 2006) and ALMA (after 2008).

Acknowledgements. The authors thank Friedrich Wyrowski, Mark Reid and Thomas Preibisch for useful discussions, and the staff of the VLA (especially Claire Chandler) for assisting with the observations.

\section{Appendix A: Flux density of an ionized accretion flow}

An H II region with a density gradient consists of an optically thick core and an optically thin envelope. The angular radius of the optically thick core $\theta_{1}$ is related to the angular radius of the $\mathrm{H}$ II region $\theta_{0}$ by

$$
\theta_{1}^{2}=2 B^{2}\left(-1+\sqrt{1+\theta_{0}^{2} / B^{2}}\right)
$$

with

$$
B=A_{\mathrm{FF}} n_{0}^{2} T_{\mathrm{e}} \theta_{0}^{2} \frac{d}{\mathrm{pc}}
$$

and

$$
A_{\mathrm{FF}}=8.235 \times 10^{-2} T_{\mathrm{e}}^{-0.35}\left(\frac{v}{\mathrm{GHz}}\right)^{-2.1} .
$$

The radio emission from a gravitationally bound $\mathrm{H}$ II region is the sum of the emissions from the optically thick core and the optically thin envelope, weighted by their respective areas on the sky. For the optically thick core, the brightness temperature is

$T_{\text {thick }}=T_{\mathrm{e}} \frac{\theta_{1}^{2}}{\theta_{0}^{2}}$

while for the optically thin envelope,

$T_{\text {thin }}=-4 A_{\mathrm{FF}} n_{0}^{2} \frac{d}{\mathrm{pc}}\left(u+\theta_{0} / 2 \ln \frac{\theta_{0}-u}{\theta_{0}+u}\right)$ 
with

$u=\sqrt{\theta_{0}^{2}-\theta_{1}^{2}}$.

If the emission is unresolved, the total flux density is

$S_{v}=2 k_{\mathrm{B}}\left(T_{\text {thick }}+T_{\text {thin }}\right) \Omega v^{2} / c^{2}$

where

$\Omega=\pi \theta_{0}^{2}$

is the solid angle of the $\mathrm{H}$ II region.

\section{References}

Aitken, D. K., Smith, C. H., James, S. D., Roche, P. F., \& Hough, J. H. 1988, MNRAS, 230, 629

Beuther, H., Schilke, P., Sridharan, T. K., et al. 2002, A\&A, 383, 892

Beuther, H., Zhang, Q., Greenhill, L. J., et al. 2004, ApJ, 616, L23

Bonnell, I. A., Bate, M. R., \& Zinnecker, H. 1998, MNRAS, 298, 93

Bunn, J. C., Hoare, M. G., \& Drew, J. E. 1995, MNRAS, 272, 346

Campbell, B. 1984, ApJ, 287, 334

Churchwell, E. 2002a, in Hot Star Workshop III: The Earliest Stages of Massive Star Birth, ed. P. A. Crowther (ASP), 3

Churchwell, E. 2002b, ARA\&A, 40, 27

Claussen, M. J., Gaume, R. A., Johnston, K. J., \& Wilson, T. L. 1994, ApJ, 424, L41

Crampton, D., Georgelin, Y. M., \& Georgelin, Y. P. 1978, A\&A, 66, 1

Fish, V. L., Reid, M. J., Argon, A. L., \& Menten, K. M. 2003, ApJ, 596, 328

Forster, J. R., \& Caswell, J. L. 1999, A\&AS, 137, 43

Gómez, Y., Rodríguez, L. F., \& Garay, G. 2002, ApJ, 571, 901

Garay, G., \& Lizano, S. 1999, PASP, 111, 1049

Gibb, E. L., Whittet, D. C. B., Schutte, W. A., et al. 2000, ApJ, 536, 347

Hasegawa, T. I., \& Mitchell, G. F. 1995, ApJ, 451, 225

Hatchell, J., \& van der Tak, F. F. S. 2003, A\&A, 409, 589

Hollenbach, D., Johnstone, D., Lizano, S., \& Shu, F. 1994, ApJ, 428, 654

Kameya, O., Morita, K., Kawabe, R., \& Ishiguro, M. 1990, ApJ, 355, 562

Keto, E. 2002a, ApJ, 568, 754

Keto, E. 2002b, ApJ, 580, 980

Keto, E. 2003, ApJ, 599, 1196

Krügel, E. 2003, The physics of interstellar dust (Bristol, UK: The Institute of Physics)

Lada, C. J., Thronson, H. A., Smith, H. A., Schwartz, P. R., \& Glaccum, W. 1984, ApJ, 286, 302

Maeder, A., \& Meynet, G. 1989, A\&A, 210, 155
Menten, K. M., \& Reid, M. J. 1995, ApJ, 445, L157

Menten, K. M., \& van der Tak, F. F. S. 2004, A\&A, 414, 289

Mitchell, G. F., \& Hasegawa, T. I. 1991, ApJ, 371, L33

Mitchell, G. F., Curry, C., Maillard, J., \& Allen, M. 1989, ApJ, 341, 1020

Mitchell, G. F., Maillard, J., Allen, M., Beer, R., \& Belcourt, K. 1990, ApJ, 363, 554

Mitchell, G. F., Maillard, J.-P., \& Hasegawa, T. I. 1991, ApJ, 371, 342

Neufeld, D. A., \& Melnick, G. J. 1991, ApJ, 368, 215

Olnon, F. M. 1975, A\&A, 39, 217

Ossenkopf, V., \& Henning, T. 1994, A\&A, 291, 943

Osterbrock, D. E. 1989, Astrophysics of gaseous nebulae and active galactic nuclei (Mill Valley, CA: University Science Books)

Ott, M., Witzel, A., Quirrenbach, A., et al. 1994, A\&A, 284, 331

Preibisch, T., Balega, Y. Y., Schertl, D., \& Weigelt, G. 2003, A\&A, 412, 735

Reid, M. J., \& Menten, K. M. 1997, ApJ, 476, 327

Reid, M. J., Argon, A. L., Masson, C. R., Menten, K. M., \& Moran, J. M. 1995, ApJ, 443, 238

Rengarajan, T. N., \& Ho, P. T. P. 1996, ApJ, 465, 363

Richling, S., \& Yorke, H. W. 1997, A\&A, 327, 317

Sandell, G., Goss, W. M., \& Wright, M. 2005, ApJ, 621, 839

Schaerer, D., \& de Koter, A. 1997, A\&A, 322, 598

Schutte, W. A., Tielens, A. G. G. M., Whittet, D. C. B., et al. 1996, A\&A, 315, L333

Tan, J. C. 2003, in Galactic Star Formation Across the Stellar Mass Spectrum, ASP Conf. Ser., 287, 207

Testi, L., Palla, F., \& Natta, A. 1999, A\&A, 342, 515

Tieftrunk, A. R., Gaume, R. A., Claussen, M. J., Wilson, T. L., \& Johnston, K. J. 1997, A\&A, 318, 931

Tofani, G., Felli, M., Taylor, G. B., \& Hunter, T. R. 1995, A\&AS, 112, 299

Torrelles, J. M., Patel, N. A., Anglada, G., et al. 2003, ApJ, 598, L115

Trinidad, M. A., Curiel, S., Cantó, J., et al. 2003, ApJ, 589, 386

Trinidad, M. A., Curiel, S., Torrelles, J. M., et al. 2004, ApJ, 613, 416

van der Tak, F. F. S. 2003, in Star Formation at High Angular Resolution, IAU Symp., 221, 59

van der Tak, F. F. S., van Dishoeck, E. F., Evans, N. J., Bakker, E. J., \& Blake, G. A. 1999, ApJ, 522, 991

van der Tak, F. F. S., van Dishoeck, E. F., Evans, N. J., \& Blake, G. A. 2000, ApJ, 537, 283

van der Tak, F. F. S., Boonman, A. M. S., Braakman, R., \& van Dishoeck, E. F. 2003, A\&A, 412, 133

van der Tak, F. F. S., Tuthill, P. G., \& Danchi, W. 2005, A\&A, 431, 993

Van Dishoeck, E. F. 1998, in Chemistry and Physics of Molecules and Grains in Space, Faraday Discussions No. 109, 31

Walmsley, C. M. 1995, Rev. Mex. Astron. Astrofis. Ser. Conf., 1, 137 Willner, S. P., Gillett, F. C., Herter, T. L., et al. 1982, ApJ, 253, 174 